\title{
Lusioersily
}

\section{Functional connectivity analysis of motor imagery EEG signal for brain-computer interfacing application}

Ghosh, P., Mazumdar, A., Bhattacharyya, S., Tibarewala, D. N., \& Hayashibe, M. (2015). Functional connectivity analysis of motor imagery EEG signal for brain-computer interfacing application. In 2015 7th International IEEE/EMBS Conference on Neural Engineering (NER) IEEE. https://doi.org/10.1109/NER.2015.7146597

Link to publication record in Ulster University Research Portal

Published in:

2015 7th International IEEE/EMBS Conference on Neural Engineering (NER)

Publication Status:

Published (in print/issue): 02/07/2015

DOI:

10.1109/NER.2015.7146597

\section{Document Version}

Author Accepted version

\section{General rights}

Copyright for the publications made accessible via Ulster University's Research Portal is retained by the author(s) and / or other copyright owners and it is a condition of accessing these publications that users recognise and abide by the legal requirements associated with these rights.

\section{Take down policy}

The Research Portal is Ulster University's institutional repository that provides access to Ulster's research outputs. Every effort has been made to ensure that content in the Research Portal does not infringe any person's rights, or applicable UK laws. If you discover content in the Research Portal that you believe breaches copyright or violates any law, please contact pure-support@ulster.ac.uk. 


\title{
Functional Connectivity Analysis of Motor Imagery EEG signal for Brain-computer Interfacing Application
}

\author{
Poulami Ghosh, Ankita Mazumder, Saugat Bhattacharyya, D.N. Tibarewala and Mitsuhiro Hayashibe
}

\begin{abstract}
The human brain can be considered as a graphical network having different regions with specific functionality and it can be said that a virtual functional connectivity are present between these regions. These regions are regarded as nodes and the functional links are regarded as the edges between them. The intensity of these functional links depend on the activation of the lobes while performing a specific task(e.g. motor imagery tasks, cognitive tasks and likewise). The main aim of this study is to understand the activation of the parts of the brain while performing three types of motor imagery tasks with the help of graph theory. Two indices of the graph, namely Network Density and Node Strength are calculated for 32 electrodes placed on the subject's head covering all the brain lobes and the nodes having higher intensity are identified.
\end{abstract}

\section{INTRODUCTION}

The main objective of any Brain-Computer Interface (BCI) system is to connect thought of a human being to the computer/machine by mapping the brain activities properly. This technology is particularly useful for providing rehabilitation to people suffering from various neural diseases like paralysis, cerebral palsy, amyotrophic lateral sclerosis and also to amputees [1]. Apart from rehabilitation, BCI has also potential applications in various other fields like communication, computer gaming, robot control. The brain signal mapping technique can either be invasive (e.g. Electrocorticography) or non-invasive (e.g. Electroencephalography). Though the invasive techniques have the ability to render better signal quality, the non-invasive techniques have some obvious advantages over them. The most common BCI systems use EEG signals for acquiring the brain signals.

The main components of such systems are feature extraction, feature selection and classification. The acquired brain signals are first preprocessed using a number of filters and then subjected to an algorithm (known as feature extraction algorithm) for extracting the features of interest from the entire dataset. The next step is basically an optional step. If the feature space is very large and contains irrelevant features then the most relevant features construct the feature vector. The last and most important component is classification of these signals. The output of the classifiers are ultimately used as command signals to drive an external devices.

Poulami Ghosh, Ankita Mazumder, D.N. Tibarewala are with School of Bioscience and Engineering, Jadavpur Uviversity, Kolkata-32, India. mazumderankita@gmail.com, pologhosh58@gmail.com, biomed. ju@gmail.com

Saugat Bhattacharyya is with School of Bioscience and Engineering, Jadavpur Univeristy, Kolkata-32, India and INRIA DEMAR Project and LIRMM, University of Montpellier, Montpellier, France. saugatbhattacharyya@ive.com

Mitsuhiro Hayashibe is with INRIA DEMAR Project and LIRMM, University of Montpellier, Montpellier, France. hayashibed lirmm.fr
In this paper we have tried to utilize functional connectivity between different parts of the human brain for classifying a motor imagery task. These functional connectivity networks can be estimated by different imaging techniques like EEG, fMRI and likewise [2]. The analysis of these networks are performed by using a very useful mathematical tool called graph theory. Graph theory basically represents the entire functional network with a number of nodes and edges between them and the amount of connectivity existing between two nodes is depicted by assigning weights to the edges between them. Brain connectivity patterns can be determined by using two types of measures, namely, Bivariate and Multivariate. Here we have considered a multivariate measure known as multivariate autoregressive (MVAR) model. One of the most widely investigated connectivity measure is the Directed Transfer Function (DTF) [3]. This function basically computes the directional influences between any two given nodes. There are a number of theoretical indices for defining a graph. In this paper, two indices, namely node strength and network density are measured from the DTF values. In the current study, the BCI competition Dataset III [4] is used for computing different multivariate measures.

This paper is organized in four sections. In section II, the materials and methods are described. Sections III, IV and V provides a brief background on Directed Transfer Function, Network Density and Node Strength. The results obtained and the inferences drawn from them are mentioned in the section VI. Section VII mentions the concluding remarks on the study mentioned in this paper.

\section{Materials And Methods}

The dataset used in this study is the BCI Competition III dataset [4]. Data is collected from three normal subjects in a relax state while performing three repetitive motor imagery tasks, which are (i)imagination of left-hand movement, (ii)imagination of right hand movement and (iii)generation of words starting with the same alphabet (tongue movement). Three values, 2,3 and 7, are assigned to the three tasks respectively. The data are collected in four sessions for all the three subjects having 4 minutes duration each and the gap between two consecutive sessions is 5-10 minutes. The subjects performed each of the tasks for 15 seconds and then switched to another task as per the instruction provided by a visual stimuli. The EEG signal acquisition system used in this experiment is a Biosemi system. In this system, the 32 electrodes(Fp1, AF3, F7, F3, FC1, FC5, T7, C3, CP1, CP5, P7, P3, Pz, PO3, O1, Oz, O2, PO4, P4, P8, CP6, CP2, C4, T8, FC6, FC2, F4, F8, AF4, Fp2, Fz, Cz), are arranged 


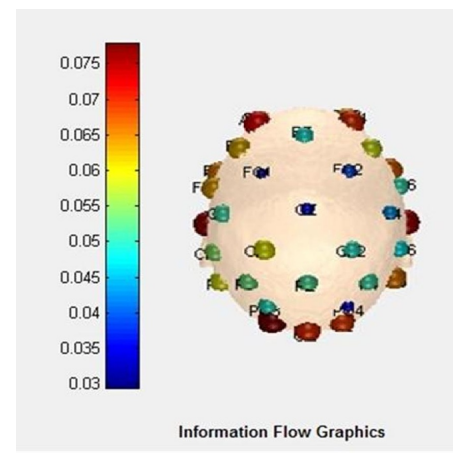

(a)

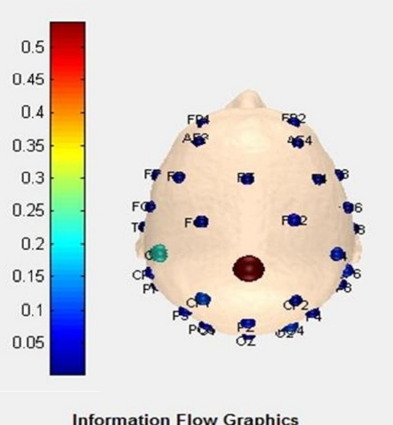

(b)

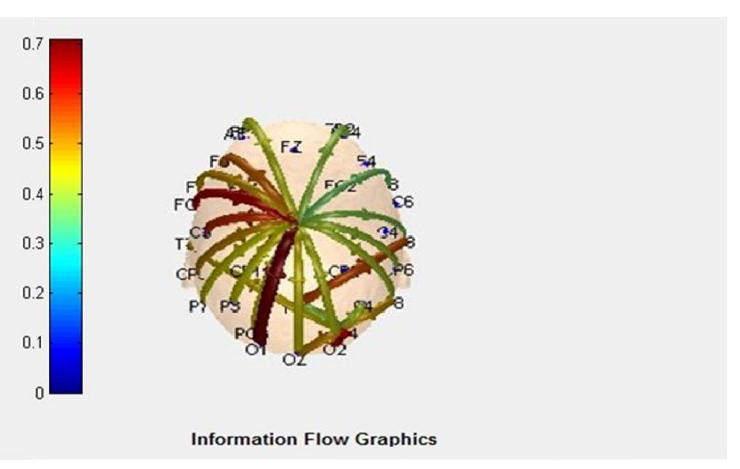

(c)

Fig. 1. (a) Inflow graph, (b) Outflow graph and (c) Out to inflow graph of the functional connectivity network of the brain while imagining right hand movement.

according to the international 10-20 electrode placement system and embedded within a cap, worn by the subjects during signal acquisition. The sampling rate of the raw EEG data acquired from the subjects is $512 \mathrm{~Hz}$. No measures were taken for rejecting any artifact or noise occurring during the experiment.

After acquisition of the data from all 32 electrodes, a matlab software named econnectome is used for generating the graphical representation of the functional connectivity network for all the three tasks. From these figures itself a primary understanding of the network could be developed. The inflow-outflow graph of subject 1 while imagining right hand movement in the first training set are given in Fig.1. Figure1(a) describes the amount of inflow of functional connectivity going out of all the 32 electrodes and these are color coded to indicate the intensity of these inflows. From Fig.1(a) it is quite evident that the inflows are maximum in the frontal, temporal and occipital lobes. Figure 1(b) depicts the functional outflow from the nodes and in contrast to Fig.1(a) it shows that the outflows are maximum from the Central lobe(Cz). In Fig 1(c), the direction of the flow between different nodes are shown and it can be seen clearly that majority of the paths are going from $\mathrm{Cz}$ to different nodes of the frontal, parietal and temporal lobes.

Apart from that, the adjacency matrices are also generated through this software which is the matrix representation of the weighted connectivity existing between different nodes. An example of such an adjacency matrix is shown in Fig.2. For this study, adjacency matrices were calculated for frequencies ranging between $8-30 \mathrm{~Hz}$ as because most of the movement related tasks EEG signals within this frequency range [5]. This adjacency matrix helps to calculate the weights of the edges of the graph with the help of Directed Transfer Function.

\section{Directed Transfer Function}

DTF is used for determination of directional influence between two nodes or in other word a pair of channels belonging to a multivariate dataset. It was first proposed by Kaminski and Blinowska in 1991 [6]. A multivariate

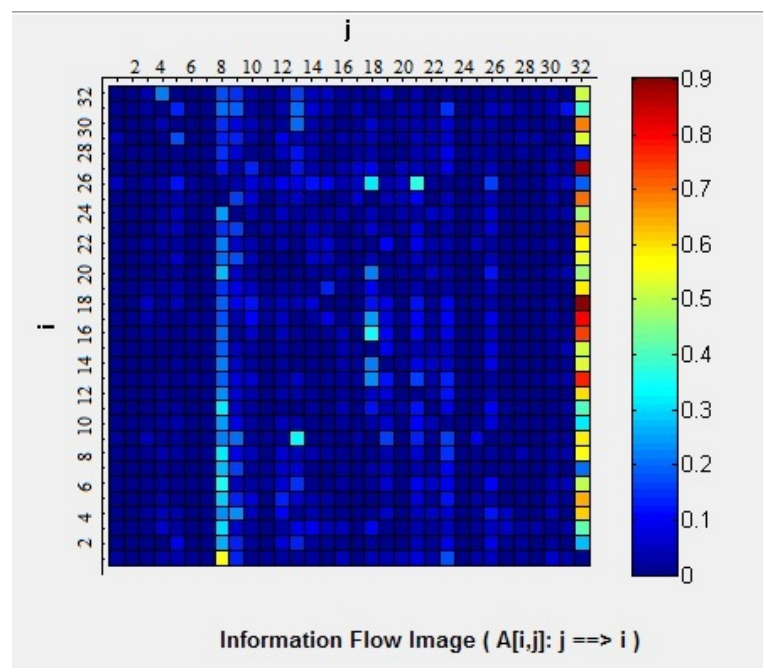

Fig. 2. Adjacency matrix $A_{i j}$.

Autoregressive model(MVAR) has to be estimated from the recorded signal for measuring the directional influence by DTF. Let us define the measurements from $\mathrm{k}$ channels at time $t$ be given by the following expression

$$
X(t)=\left[X_{1}(t), X_{2}(t), \ldots X_{k}(t)\right]^{T}
$$

where the T denotes transpose of matrix. Again let us assume that an MVAR process can describe $X(t)$ properly and given as

$$
X(t)=\sum_{i=1}^{p} A(i) X(t-i)+E(t)
$$

When converted to the frequency domain, this equation is written as,

$$
A(f) X(f)=E(f)
$$

In the above equation, $\mathrm{A}(\mathrm{f})$ can be written as,

$$
A(f)=-\sum_{j=0}^{p} A(i) e^{-2 \pi f j}
$$




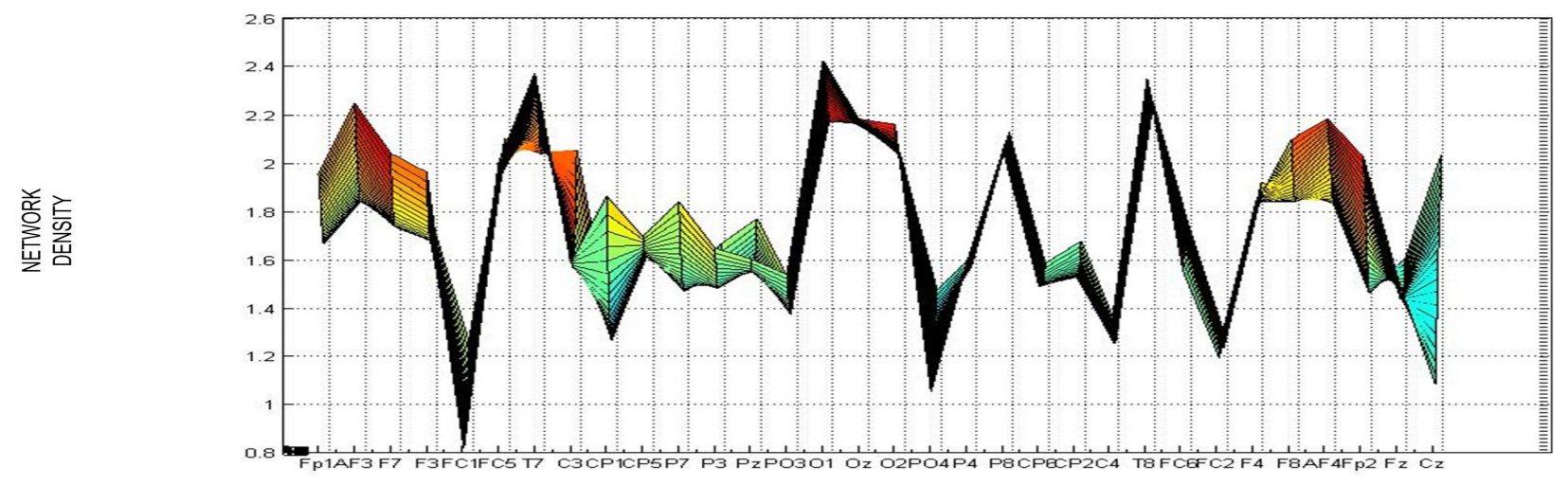

ELECTRODES

Fig. 3. Network Densities of 32 electrodes while performing the motor imagery task for tongue.

From (4) it can be obtained that $\mathrm{A}(0)=-\mathrm{I}$, where I denotes the identity matrix. As a result, (3) can also be written as,

$$
X(f)=A^{-1}(f) E(f)
$$

Here, $A^{-1}(f)$ can be written as $\mathrm{H}(\mathrm{f})$ which is basically the transfer matrix of the system. Finally, the directional influence or DTF can be given as, $\left|H_{i j}\right|^{2}$. The weights of the links between two nodes ( $i$ and $j$ ) is calculated from this directional influence.

\section{NeTwork Density}

The simplest parameter of any graphical network is the density of this network. It is defined as the ratio of the number of connections or channels present in a network and its maximum capacity [7]. Graphs can be either weighted or non-weighted. In case of non-weighted graphs the value of the network density ranges between 0 and 1 . This index basically gives an idea of the overall level of connectivity of the entire network. The network density of the weighted graph can be calculated from the following equation

$$
D=\sum_{i \neq j \in N} w_{i j}
$$

where $w_{i j}$ denotes the weight of the edge from $\mathrm{i}$ to $\mathrm{j}$ and $\mathrm{N}$ is the set of all the nodes.

\section{NOde StREnGth}

It is basically an attribute which defines the amount of connections a node has with other vertices of the graph [8]. A node can have two types of strengths viz. in and out strength, given by $S_{\text {in }}$ and $S_{\text {out }}$ respectively. This is applicable only for directed graphs. These two parameters take two things into consideration, namely, the number of nodes connected to that specific node and also the weights of the connections existing between them. $S_{i n}$ measures the total amount of functional inflow received by a particular node 'i' and can be calculated using the following equation

$$
S_{i n}=\sum_{j \in N} w_{i j}
$$

On the contrary, the measure $S_{\text {out }}$ actually gives the measure of the total functional outflow from a particular node 'i' and is given by

$$
S_{\text {out }}=\sum_{j \in N} w_{j i}
$$

\section{Results AND Discussions}

The main aim of this paper is to investigate two parameters of the functional connectivity network of the brain while performing motor imagery task. In order to calculate these parameters, the fundamental prerequisites are the values of the weights of the graph or in other words, the intensity of the functional connectivity between the different regions of the brain. This is achieved by calculating DTF matrices with the help of Econnectome.

After obtaining the weights, Network Densities for all the electrodes over the entire range of frequencies $(8-30 \mathrm{~Hz})$ are calculated. Fig. 3 represents the network density of the nodes or electrodes while generating words starting with the same alphabet i.e. while performing the motor imagery task for tongue during the second training session. It can be concluded from Fig. 3 that the network densities are very high in case of some of the electrodes in comparison to other nodes and as a result, these regions have higher intensity of network connectivity. We have considered another parameter in this study, known as the node strength. As mentioned before, both of the inflow and the outflow strengths needed to be measured. These strengths are calculated using (7) and (8) respectively for all the subjects while executing the three types of motor imagery tasks during all the training sessions and then these values of are plotted for all the nodes from which the EEG signal was acquired. This plot is shown in Fig.4 and Fig.5. If both of these figures are compared, it can be easily concluded that the overall strength of the nodes are higher in case of Sin and the in-strength of the temporal, frontal and parietal lobes are much higher than the central region, whereas, a completely reverse trend can be observed in case of out-strength. There, the central lobe 


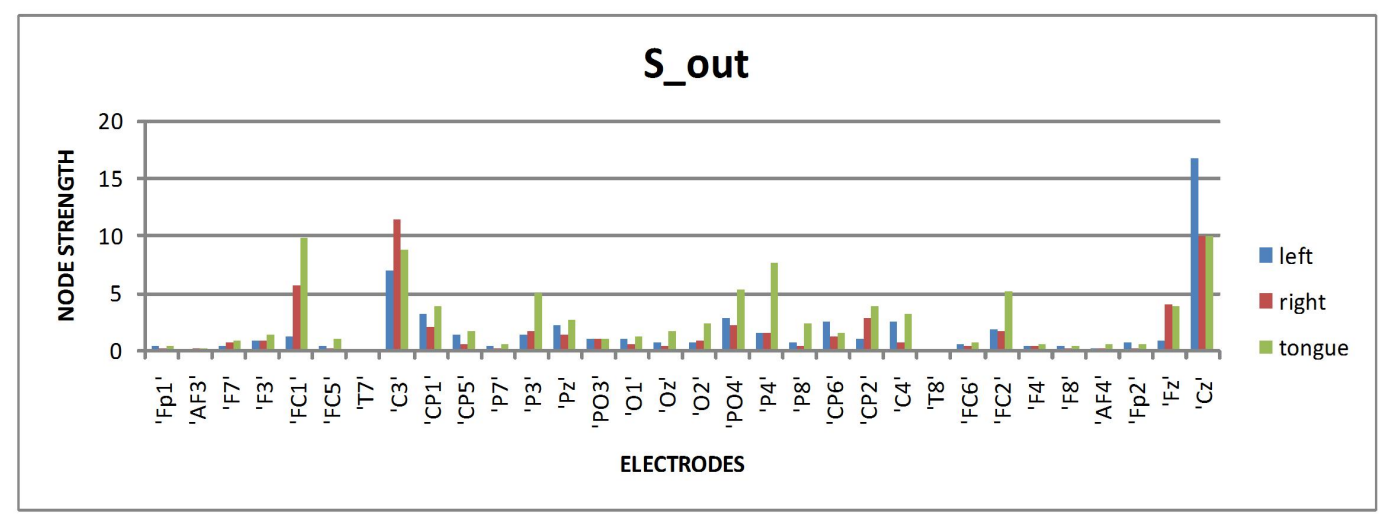

Fig. 4. $S_{\text {out }}$ of all the nodes while performing all the three motor imagery tasks.

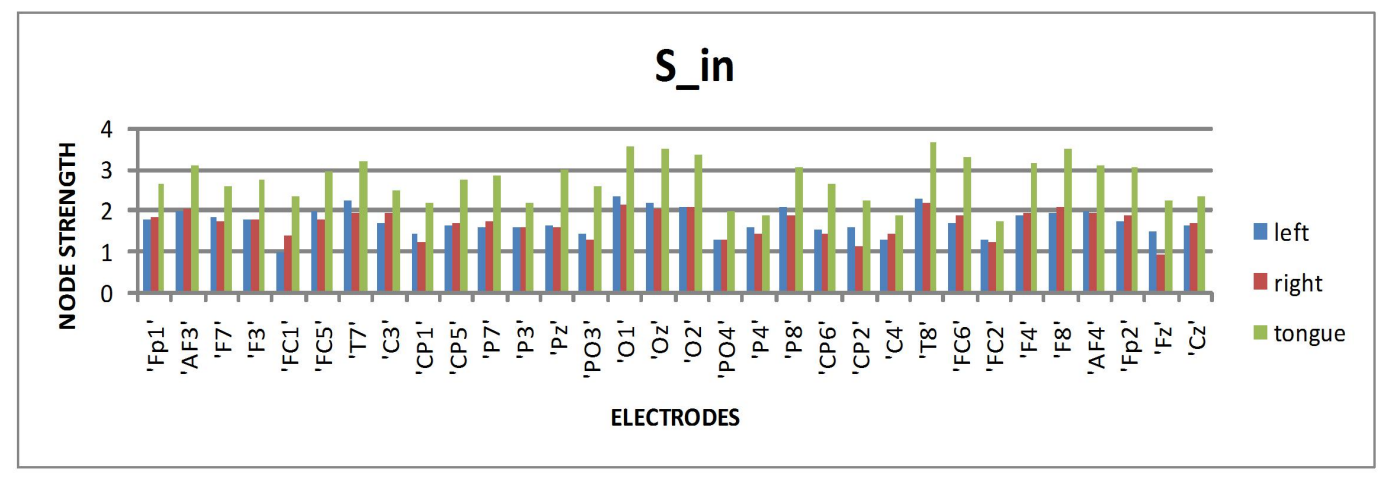

Fig. 5. $S_{i n}$ of all the nodes while performing all the three motor imagery tasks.

electrodes(especially $\mathrm{Cz}$ ) is very strong. This inference is quite similar to the inferences drawn from Fig.1.

Another important inference can be drawn from Fig.4 is that, if the data for the left and right hand movement are considered only, it can be seen that the out-strength for the node $\mathrm{C} 3$ is higher for right hand movement and that of node $\mathrm{C} 4$ is higher for node $\mathrm{C} 3$. It is a well-established fact that the left portion of the brain controls the movements of the right hand side and vice versa [9] and the graph in Fig.4 confirms that. So, it may also be possible for classifying the left and right hand movement without even using classification algorithm. As a result, this graph theoretical approach can have huge application in BCI systems as classifier.

\section{CONCLUSION}

The results obtained from the study revealed that the functional outflow while performing motor imagery tasks are maximum at the nodes placed on central lobe but the inflow is much stronger at Occipital, Parietal and Temporal lobes. It is concluded that from the graphs that the left and right limb movement tasks could be classified with the help of Node Strength $\left(S_{\text {out }}\right)$ and without using any classification algorithm. Another aspect that was noticed is that, for both of the parameters, the intensities are highest for tongue movement task in comparison to other two tasks. The similar trend was noted in almost all the training sessions of the three subjects.

\section{REFERENCES}

[1] J. Daly, and J.R. Wolpaw, "Brain-computer interfaces in neurological rehabilitation," Lancet Neurol., vol. 7, no. 11, pp. 10321043, 2008.

[2] F. Babiloni, F. Cincotti, C. Babiloni, F. Carducci, D. Mattia, L. Astolfi, "Estimation of the cortical functional connectivity with the multimodal integration of high-resolution EEG and fMRI data by directed transfer function," Neuroimage, vol.24, no. 1, pp. 118-131, 2005.

[3] M.Kamiski, M. Ding, W. A. Truccolo, S. L. Bressler, "Evaluating causal relations in neural systems: Granger causality, directed transfer function and statistical assessment of significance," Biological cybernetics, vol.85, no. 2, pp. 145-157, 2001.

[4] M. J. Kaminski, K. J.Blinowska,"A new method of the description of the information flow in the brain structures," Biological cybernetics, vol.65, no. 3, pp. 203-210, 1991.

[5] J. R. del Milln, "On the need for on-line learning in brain-computer interfaces," In: Proc. Neural Networks, IEEE International Joint Conference, Vol. 4, pp. 2877-2882, July 2004.

[6] G. Pfurtscheller, C. Neuper,"Motor imagery and direct brain-computer communication," In: Proc. of the IEEE, vol. 89, no. 7, pp. 1123-1134, 2001.

[7] F. De Vico Fallani, L. Astolfi, F. Cincotti, D. Mattia, A. Tocci, S. Salinari, F. Babiloni, "Brain network analysis from high-resolution EEG recordings by the application of theoretical graph indexes," Neural Systems and Rehabilitation Engineering, IEEE Transactions, vol. 16, no. 5, pp. 442-452, 2008.

[8] F. D. Fallani, L. F. Costa, F. A. Rodriguez, L. Astolfi, G. Vecchiato, J. Toppi, F. Babiloni, "A graph-theoretical approach in brain functional networks. Possible implications in EEG studies," Nonlinear biomedical physics, vol. 4,Suppl. 1, S8, 2010.

[9] A. R. Luft, G. V. Smith, L. Forrester, J. Whitall, R. F. Macko, T. K. Hauser, D. F Hanley, "Comparing brain activation associated with isolated upper and lower limb movement across corresponding joints." Human brain mapping, vol. 17 no. 2, pp. 131-140, 2002. 